\title{
Dimethyloxaloylglycine increases bone repair capacity of adipose-derived stem cells in the treatment of osteonecrosis of the femoral head
}

\author{
ZHEN-HONG ZHU, WEN-QI SONG, CHANG-QING ZHANG and JI-MIN YIN \\ Department of Orthopedic Surgery, Sixth People's Hospital Affiliated to Shanghai Jiao Tong University, \\ Shanghai 200233, P.R. China
}

Received June 21, 2015; Accepted August 11, 2016

DOI: $10.3892 /$ etm.2016.3698

\begin{abstract}
Mesenchymal stem cells have been widely studied to promote local bone regeneration of osteonecrosis of the femoral head $(\mathrm{ONFH})$. Previous studies observed that dimethyloxaloylglycine (DMOG) enhanced the angiogenic and osteogenic activity of mesenchymal stem cells by activating the expression of hypoxia inducible factor- $1 \alpha$ (HIF-1 $\alpha)$, thereby improving the bone repair capacity of mesenchymal stem cells. In the present study, it was investigated whether DMOG could increase the bone repair capacity of adipose-derived stem cells (ASCs) in the treatment of ONFH. Western blot analysis was performed to detect HIF-1 $\alpha$ protein expression in ASCs treated with different concentrations of DMOG. The results showed DMOG enhanced HIF-1 $\alpha$ expression in ASCs in a dose-dependent manner at least for 7 days. Furthermore, DMOG-treated ASCs were transplanted into the necrotic area of a rabbit model of ONFH to treat the disease. Four weeks later, micro-computed tomography (CT) quantitative analysis showed that $58.8 \pm 7.4 \%$ of the necrotic area was regenerated in the DMOG-treated ASCs transplantation group, $45.5 \pm 3.4 \%$ in normal ASCs transplantation group, $25.2 \pm 2.8 \%$ in only core decompression group and $10.6 \pm 2.6 \%$ in the untreated group. Histological analysis showed that transplantation of DMOG-treated ASCs clearly improved the bone regeneration of the necrotic area compared with the other three groups. Micro-CT and immunohistochemical analysis demonstrated
\end{abstract}

Correspondence to: Dr Ji-Min Yin, Department of Orthopedic Surgery, Sixth People's Hospital Affiliated to Shanghai Jiao Tong University, 600 Yishan Road, Shanghai 200233, P.R. China

E-mail: yinjimin123@163.com

Abbreviations: MSCs, mesenchymal stem cells; HIF-1 $\alpha$, hypoxia inducible factor-1 $\alpha$; DMOG, dimethyloxalylglycine; ASCs, adipose-derived stem cells; ONFH, osteonecrosis of the femoral head

Key words: adipose-derived stem cells, bone repair, dimethyloxaloylglycine, hypoxia inducible factor- $1 \alpha$, osteonecrosis of the femoral head the revasculation of the necrotic area were also increased significantly in the DMOG-treated ASC group compared with the control groups. Thus, it is hypothesized that DMOG could increase the bone repair capacity of ASCs through enhancing HIF-1 $\alpha$ expression in the treatment of ONFH.

\section{Introduction}

Osteonecrosis of the femoral head (ONFH) is a pathological process primarily caused by interrupted local blood circulation, which can cause apoptosis of osteocytes and osseous tissue necrosis $(1,2)$. The lack of effective therapy for ONFH is a difficulty that needs to be overcome in clinical practice. Several methods have been used to treat early-stage ONFH, such as drug therapy, core decompression, vascularized bone grafting and rotational osteotomy. However, the clinical results of these methods are not satisfactory $(3,4)$.

Previous studies have described the use of mesenchymal stem cells (MSCs) to promote local bone repair and healing $(5,6)$. MSCs are pluripotent and can differentiate into several lineages of cells, which have shown potency in the treatment of numerous ischemic diseases, such as myocardial infarction $(7,8)$, nerve injury (9) and bone defect (10). Prior studies have found that the implantation of MSCs into the necrotic area of the femoral head was able to improve local bone regeneration $(11,12)$. MSCs are able to differentiate into osteoblasts to promote local bone healing directly $(13,14)$. Furthermore, MSCs are able to secrete growth factors such as vascular endothelial growth factor (VEGF), angiopoietin 1, stromal cell-derived factor 1 and basic fibroblast growth factor, which have angiogenic potency and can promote local revascularization, thereby improving bone healing indirectly $(15,16)$.

Hypoxia inducible factor- $1 \alpha(\mathrm{HIF}-1 \alpha)$ is a key mediator of the adaptive cell response to hypoxia, which controls the expression of numerous genes and modulates cell proliferation, differentiation and pluripotency (17). Previous experiments have suggested that HIF-1 $\alpha$ could enhance the osteogenic differentiation of MSCs and the expression of angiogenic factors, thus promoting their bone healing capacity (18). In a prior study, we implanted HIF-1 $\alpha$ transgenic MSCs into the necrotic area of the femoral head, and found that this treatment resulted in improved osteogenic and angiogenic capacity 
in vitro and in vivo, leading to better results for early-stage ONFH (19). However, the risks of lentivirus vectors, such as tumorigenesis (20), should be seriously considered before clinical application.

Dimethyloxalylglycine (DMOG) is a cell permeable prolyl-4-hydroxylase inhibitor, which is able to stabilize expression of HIF-1 $\alpha$ in cells at normal oxygen tension (21). Therefore, DMOG is hypothesized to be an alternative strategy for enhancing HIF-1 $\alpha$ expression in MSCs. In a previous study, we demonstrated that DMOG could increase HIF-1 $\alpha$ expression in MSCs, accordingly enhancing their bone healing capacity (22). In the present study, we investigated whether DMOG was able to enhance the bone repair capacity of adipose-derived stem cells (ASCs) in treating early-stage ONFH.

\section{Materials and methods}

Animals. Healthy New Zealand rabbits weighing 2.5-3 kg and aged 2-3 months were provided by the Experimental Animal Centre of Shanghai Jiao Tong University affiliated Sixth People's Hospital (Shanghai, China). Animals were maintained in single cages at a controlled temperature $\left(15-25^{\circ} \mathrm{C}\right)$ under a 12-h light/dark cycle and fed with a standard diet. Animals received humane care in compliance with the Guide of the US Department of Health for the care and use of laboratory animals (23). The experiment protocol was approved by the Animal Ethics Committee of Shanghai Jiao Tong University.

Isolation and culture of ASCs. Primary ASCs were harvested from the adipose tissue of New Zealand rabbits. Briefly, the animals were anesthetized with pentobarbital sodium (3 mg/100 g; Sigma-Aldrich; Merck KGaA, Darmstadt, Germany). Adipose tissues were harvested and digested with $0.1 \%$ collagenase I (Sigma-Aldrich) for $1 \mathrm{~h}$. The complex was filtered with a $100-\mu \mathrm{m}$ nylon mesh (Shanghai Bolting Cloth Manufacturing Co., Ltd., Shanghai, China) and centrifuged at room temperature for $30 \mathrm{~min}$ at $363 \mathrm{x} \mathrm{g}$ The cells were then resuspended with Dulbecco's modified Eagle's medium (Gibco; Thermo Fisher Scientific, Inc., Grand Island, NY, USA) supplemented with $10 \%$ fetal bovine serum (Invitrogen; Thermo Fisher Scientific, Inc., Carlsbad, CA, USA), and were plated on culture flask (Corning Life Sciences, Tewksbury, MA, USA). The cells were cultured at $37^{\circ} \mathrm{C}$ in a humidified $5 \%$ $\mathrm{CO}_{2}$ incubator. The culture medium was replaced every 3 days, and non-adherent cells were removed. The cells were passaged approximately at a 1:3 split at subconfluence. The cells of four to six passages were used for the following experiments.

Western blot analysis. To evaluate the influence of DMOG (Sigma-Aldrich) on the expression of HIF-1 $\alpha$ protein in ASCs, the cells were seeded on six-well plates at $3 \times 10^{5}$ cells/well and regular medium (Dulbecco's modified Eagle's medium supplemented with $10 \%$ fetal bovine serum) was added with different concentrations of DMOG $(0,200,500$ and 1,000 $\mu \mathrm{M})$. After 1,3 and 7 days, total protein was harvested from the cultured cells according to standard protocols (24). Briefly, cells were washed three times with (PBS; Sinopharm Chemical Reagent Co., Ltd., Shanghai, China), and then solubilized in lysis buffer (Thermo Fisher Scientific, Inc., Waltham, MA, USA) at $4^{\circ} \mathrm{C}$ for
$10 \mathrm{~min}$. Lysates were centrifuged at $14,000 \mathrm{x} g$ for $15 \mathrm{~min}$. The supernatants were collected and stored at $-80^{\circ} \mathrm{C}$. The protein concentration was measured using a bicinchoninic protein assay kit (Thermo Fisher Scientific, Inc.). Proteins (10 $\mu \mathrm{g})$ from each sample were then separated using 12\% SDS-PAGE (Bio-Rad Laboratories, Inc., Hercules, CA, USA) and transferred to nitrocellulose membranes. They were then blocked in 5\% non-fat milk at room temperature for $1 \mathrm{~h}$. Primary antibodies against HIF-1 $\alpha$ (1:800; ab1; Abcam, Cambridge, MA, USA) were added to incubate with the membranes at $4^{\circ} \mathrm{C}$ overnight. Then, infrared-conjugated secondary antibodies (1:10,000; ab97040; Abcam) were added to incubate with the membranes for $1 \mathrm{~h}$ at room temperature. The resulting membranes were scanned in an Odyssey Scanner (Li-COR Biosciences, Lincoln, NE, USA), and quantified using Odyssey software version 3.0. The protein levels were normalized against those of $\beta$-actin (1:500; ab6276; Abcam).

Preparation of transplanted composite. A self-assembling peptide gel (BeaverNano ${ }^{\mathrm{TM}}$ hydrogel; Cyagen Biosciences, Inc., Guangzhou, China) was used as the scaffold for loading ASCs. The transplanted composite was prepared by mixing cells with the hydrogels according to the manufacturer's protocol. In brief, $1 \times 10^{7}$ ASCs were suspended in $1 \mathrm{ml} 10 \%$ sucrose solution (Sinopharm Chemical Reagent Co., Ltd.), and the cell suspension was mixed with the hydrogels in an equal volume. Then, PBS was slowly added to the mixed hydrogel composites in an equal volume and incubated for $30 \mathrm{~min}$ at room temperature to allow cross-linking. For the DMOG-treated ASCs, cells were cultured in regular medium with $1,000 \mu \mathrm{M}$ DMOG for $24 \mathrm{~h}$ prior to being mixed with the hydrogel. To ensure ASCs were continuously exposed to DMOG after implantation into the femoral head, 1,000 $\mu \mathrm{M}$ DMOG was added to PBS during the composite mixing procedure.

Animal ONFH model and treatment protocol. A total of 50 New Zealand rabbits (age, 2-3 months) were used to established ONFH models according to previously reported protocols (25). In brief, one injection of lipopolysaccharide (LPS; $10 \mu \mathrm{g} / \mathrm{kg}$; Sigma-Aldrich) was administered intravenously at day 1 , then three injections of methylprednisolone (MPS; 20 mg/kg; Pfizer, Inc., New York, NY, USA) were administered intramuscularly at days 2, 3 and 4. Six rabbits died of the inductive protocol after the injection. ONFH was confirmed using magnetic resonance imaging (MRI; GE Healthcare, Chicago, IL, USA) at 6 weeks after the last injection of MPS. The stage of ONFH was evaluated respectively by two experienced radiologists in a blinded fashion. Then the rabbits with early-stage ONFH were randomly divided into four groups: i) Group I ( $n=11)$ did not receive any therapy and served as controls; ii) Group II $(n=11)$ only received core decompression of the femoral head; iii) Group III $(n=11)$ received core decompression and normal ASC transplantation; and iv) Group IV ( $\mathrm{n}=11)$ received core decompression and DMOG-treated ASC transplantation.

Surgical procedure and perfusion. The surgical procedure was performed as previously reported (26). In brief, the animals were anesthetized with intravenous pentobarbital, and a lateral approach was made to expose the greater trochanter 
under aseptic conditions. A drill with an diameter of $1 \mathrm{~mm}$ was inserted at the flare of the greater trochanter and into the necrotic area of the femoral head, assisted by a C-arm X-ray machine. The necrotic tissue was then removed completely, and the cell-hydrogel composites were transplanted into the necrotic area through the bone tunnel made by drill for Groups III and IV. The bone tunnel was sealed with an absorbable collagen sponge plug, and the wound was closed in layers. Following the operation, animals received gentamicin (80 MU/day; Shanghai No.1 Biochemical \& Pharmaceutical Co., Ltd., Shanghai, China) intramuscularly as prophylaxis for 3 days, and all animals were free to move.

Four weeks after the surgery, animals were anesthetized with intravenous pentobarbital. The abdominal cavity of the rabbits was opened. A syringe needle was inserted in the abdominal aorta distal to the heart, and the abdominal aorta proximal to the heart was ligated. Then the abdominal vein was cut open, and heparinized normal saline (Shanghai No.1 Biochemical \& Pharmaceutical Co., Ltd.) was injected in the vasculature through the needle at a flow speed of $\sim 20 \mathrm{~mm} / \mathrm{min}$. When the outflow was limpid, a silicone injection compound (MICROFIL MV-122; Flow Tech, Inc., Carver, MA, USA) was pumped into the vasculature of the femoral head. The animals were then stored at $4^{\circ} \mathrm{C}$ to ensure polymerization of the contrast agent. After $1 \mathrm{~h}$, the proximal parts of the femurs were harvested and fixed in $4 \%$ paraformaldehyde.

Micro-computed tomography (CT) scanning. To evaluate bone regeneration in the necrotic area of the femoral head, the samples were scanned with micro-CT. The scan was performed using a micro-CT scanner (SkyScan 1076; Bruker micro-CT, Kontich, Belgium) at a resolution of $25 \mu \mathrm{m}$ and with the following settings: Anode current, $450 \mu \mathrm{A}$; X-ray voltage, $80 \mathrm{KVp}$; and exposure time, $400 \mathrm{msec}$. Bone tissue was defined at the threshold of 800 Hounsfield units (HU). The necrotic area of the femoral head was selected as the region of interest (ROI) with the aid of preoperative MRI. The parameters of bone volume/total volume (BV/TV) and bone mineral density (BMD) of ROI were calculated, which indicated the bone regeneration of the necrotic area.

These samples were then decalcified with $10 \%$ ethylenediaminetetraacetic acid (Sinopharm Chemical Reagent Co., Ltd., Shanghai, China) at $37^{\circ} \mathrm{C}$ for 3 weeks. Following decalcification, the samples underwent micro-CT examination again at a resolution of $18 \mu \mathrm{m}$ per voxel to evaluate the vascularization of the necrotic area. For segmentation of vessels from background, noise was removed with a low pass Gaussian filter and vessels were defined at the threshold of $85 \mathrm{HU}$. To reconstruct the three-dimensional architecture of blood vessels in the necrotic area, the vessels were included at each two-dimensional section by built-in 'Contouring Program' for automatic reconstruction of three-dimensional images. The axial slices through the samples were visualized, and the volume of the vessels in the necrotic area was calculated.

Histology and immunohistochemistry. After micro-CT scanning, samples were dehydrated and made transparent using dimethylbenzene (Sinopharm Chemical Reagent Co. Ltd.). They were then embedded in wax and sectioned into $6 \mu \mathrm{m}$ coronal planes. Sections were stained with hematoxylin and eosin (H\&E) and observed under a light microscope (Leica Microsystems GmbH, Wetzlar, Germany). Image-Pro Plus 6.0 software (Media Cybernetics, Inc., Rockville,MD, USA) was used to evaluate new bone formation at x100 magnification in six randomly selected fields per section. Bone density was defined as the ratio of new bone area to total area. The border of the new bone and osteoid tissue was difficult to define, so osteoid tissue was not included in new bone calculations.

Immunohistochemistry was performed using antibodies specific for CD31 (1:200; ab24590; Abcam) and HIF-1 $\alpha$ (1:100; ab8366; Abcam). In brief, these sections were rehydrated and incubated with primary antibodies at $4^{\circ} \mathrm{C}$ overnight, then incubated with biotinylated secondary IgGs (1:500; BA1001; Wuhan Boster Biological Technology, Ltd., Wuhan, China). Sections were treated with ABC complex and developed with 3,3'-diaminobenzidine (both Wuhan Boster Biological Technology, Ltd.), then stained with hematoxylin. All sections were consistently maintained in liquid, and sections incubated without primary antibodies were used as a control.

Statistical analysis. Data are expressed as the mean \pm standard deviation. One-way analysis of variance with an StudentNewman-Keuls post hoc analysis was applied to determine statistical significance. $\mathrm{P}<0.05$ was considered to indicate a statistically significant difference. Statistical analysis was performed using SPSS software, version 12.0 (SPSS, Inc., Chicago, IL, USA).

\section{Results}

HIF-1 $\alpha$ overexpression in rabbit ASCs. Western blot analysis was performed to detect the protein expression of HIF-1 $\alpha$ in rabbit ASCs treated with different concentrations of DMOG. The data showed the expression of HIF-1 $\alpha$ protein was increased in response to DMOG treatment in a dose-dependent manner (Fig. 1). After treatment for 1 day, the HIF-1 $\alpha$ expression in cells respectively increased by 2 -, 4- and 5-fold for 200, 500 and 1,000 $\mu \mathrm{M}$ DMOG, respectively, compared with the untreated ASCs. After treatment for 7 days, the levels of HIF-1 $\alpha$ protein in ASCs had no significant difference compared with ASCs treated with DMOG for 1 days, which indicated DMOG could enhance HIF-1 $\alpha$ expression at least for 7 days.

Assessment of bone regeneration in the necrotic area. Bone regeneration in the necrotic area of each group was initially analyzed using micro-CT scanning. Bone microarchitecture of each group was reconstructed in three dimensions for presentation (Fig. 2A). The samples of Group I, which received no treatment, showed few destroyed trabeculae in the necrotic area of the femoral head. In Group II, which received core decompression, the trabeculae in the necrotic area was thin and sparse. In Group III, which received core decompression and transplantation of normal ASCs, the trabeculae in the necrotic area was more intact. In Group IV, which received core decompression and transplantation of DMOG treated ASCs, the trabeculae in the necrotic area appeared intact and well distributed. Quantitative analysis indicated nearly no new bone formation in the necrotic area of Group I (Fig. 2B). By contrast, $\sim 25.2 \pm 2.8 \%$ of the necrotic area was regenerated in 
A

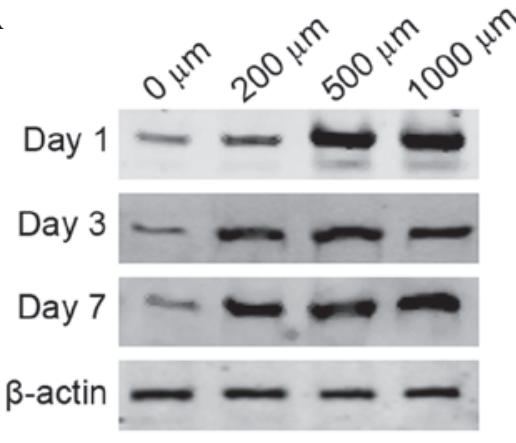

B

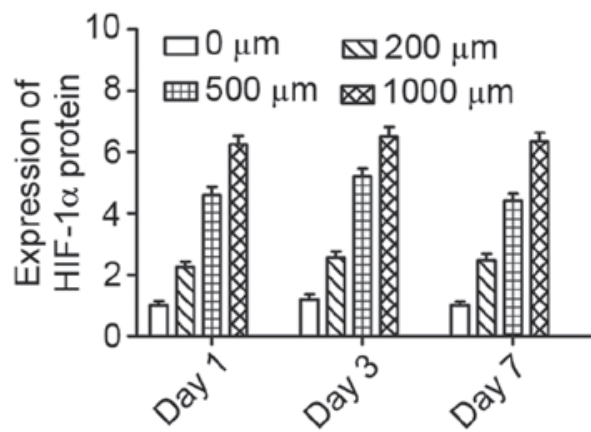

Figure 1. Effect of DMOG on the protein expression of HIF-1 $\alpha$ in ASCs. (A) Western blot analysis showed that DMOG increased the (B) protein levels of HIF-1 $\alpha$ in ASCs in a dose-dependent manner at least for 7 days. DMOG, dimethyloxalylglycine; ASCs, adipose-derived stem cells; HIF-1 $\alpha$, hypoxia inducible factor-1 $\alpha$.

A

Group I
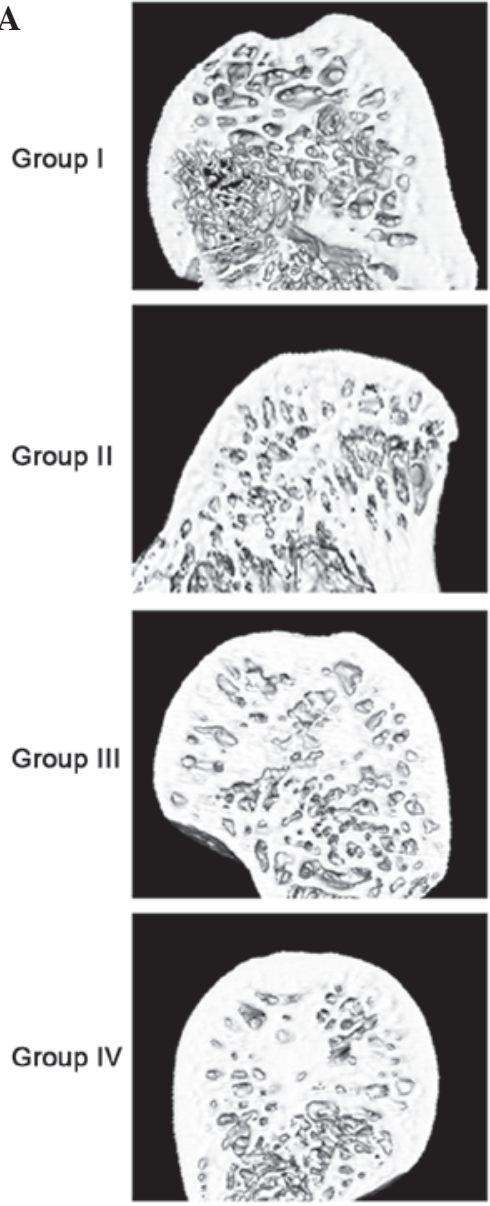

B
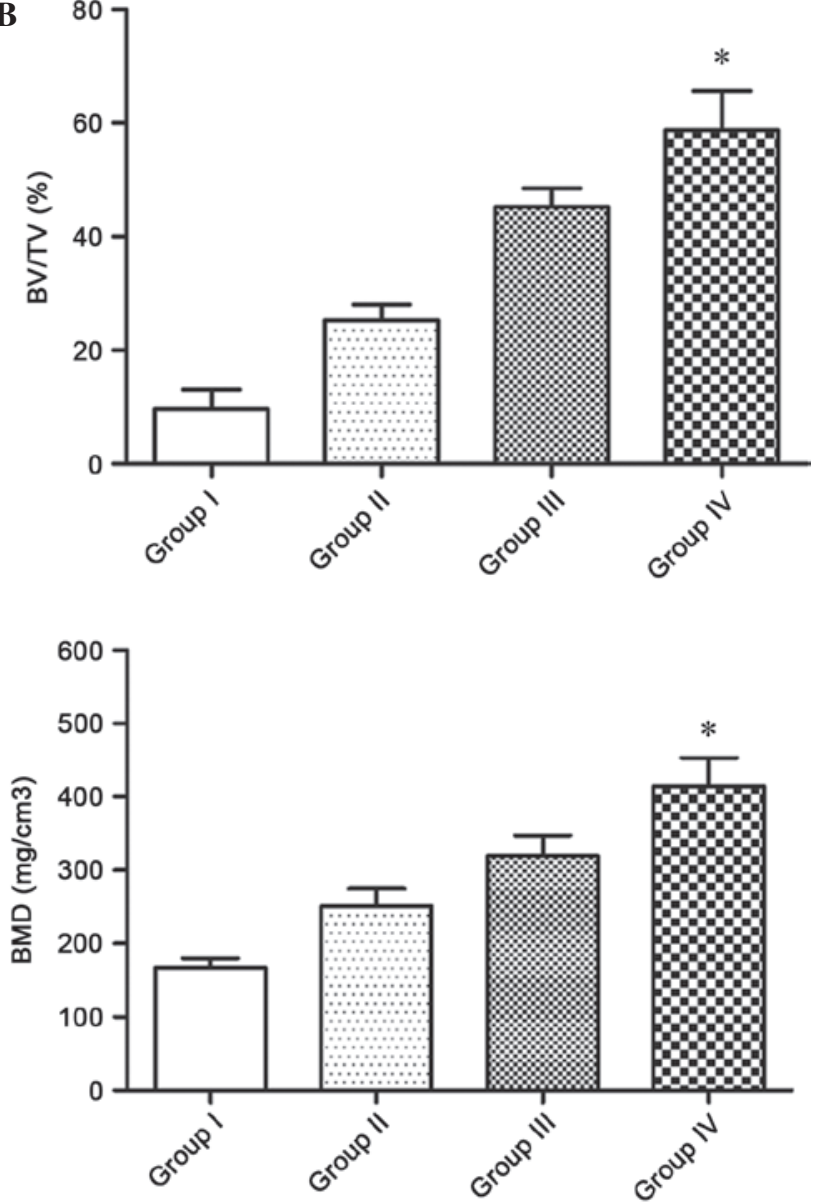

Figure 2. Micro-CT analysis of bone regeneration in the necrotic area of the femoral head. (A) Micro-CT images of the femoral head taken 4 weeks after cell implantation. (B) Morphometric analysis showed that BMD and BV/TV of the necrotic area in Group IV was significantly higher than in the other three groups $($ ( $\mathrm{P}<0.05)$. CT, computed tomography; BV, bone volume; TV, total volume; BMD, bone mineral density.

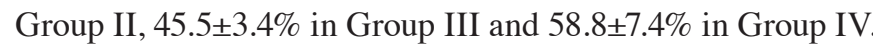
The BMD of the new bone in the necrotic area of Group IV was also significantly higher than that of other three groups $(\mathrm{P}<0.05)$.

Bone regeneration in the necrotic area of each group was also evaluated using H\&E staining. In Group I, there were numerous fat cells and rare trabecular tissue in the necrotic area, and numerous empty lacunae distributed along the trabeculae (Fig. 3A). In Group II, there was less granulation tissue and fewer fat cells in the necrotic area, and some empty lacunae were observed (Fig. 3B). In Group III, there was some disordered trabecular tissue in the necrotic area, which was obviously more than in Group II (Fig. 3C). In Group IV, there was substantial trabecular tissue, and large osteocytes were distributed along the trabeculae (Fig. 3D). Histomorphometric analysis confirmed that new bone in Group IV was more than that of other three groups, which was consistent with the micro-CT data $(\mathrm{P}<0.05$; Fig. $3 \mathrm{E})$. 


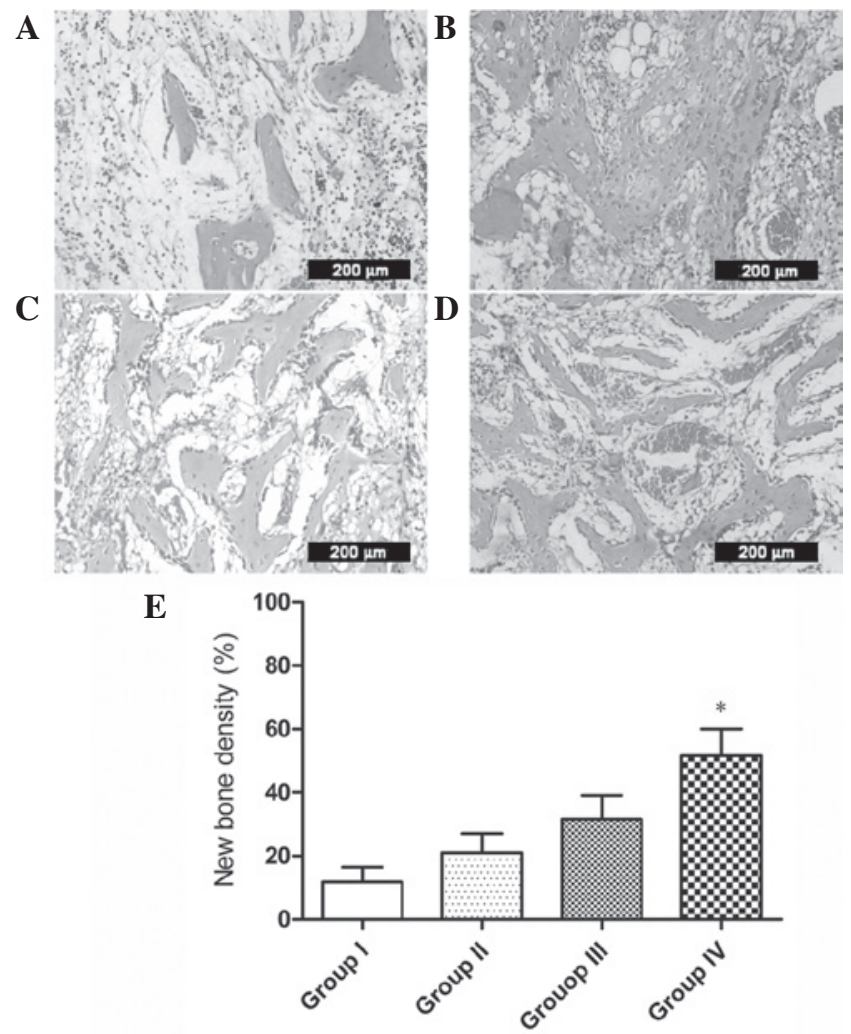

Figure 3. Histological evaluation of bone regeneration in the necrotic area of various groups. Representative histological photomicrographs of the necrotic area in groups (A) I, (B) II, (C) III and (D) IV. (E) Histomorphometric analysis showed that the bone regeneration in the necrotic area of Group IV was significantly increased compared with that in the other three groups $(\mathrm{P}<0.05)$.

Assessment of neovascularization in the necrotic area. The vascularization in the necrotic area of each group at four weeks after surgery was evaluated by perfusing the blood vessels with a silicone injection compound and imaging with micro-CT. The reconstructed three dimensional images showed the morphology of regenerated vessels in the necrotic area. In Group I, there was nearly no vascular architecture in the necrotic area (Fig. 4A). In Group II, only some blood vessels were observed (Fig. 4B). In Group III, the density of the vessels was significantly higher than that of Group II (Fig. 4C). In Group IV, there were large vessels, which were obviously increased compared to the other three groups (Fig. 4D). Quantitative analysis also showed that the average number and volume of blood vessels penetrating in the necrotic area of Group IV were significantly more than the other three groups $(\mathrm{P}<0.05$; Fig. 4E). In addition, immunohistochemistry for CD31 was performed to evaluate newly formed vessels in the necrotic area. Blood vessels were defined with positive CD31 stain and their typical round or oval structure. The results showed more vessels in Group IV than the other three groups, which supported the micro-CT results (Fig. 5).

HIF-1 $\alpha$ expression in ASCs in vivo. Immunohistochemistry for $\mathrm{HIF}-1 \alpha$ was performed to evaluate HIF-1 $\alpha$ expression in ASCs in vivo (Fig. 5). In Groups I, II and III, there were no obvious HIF-1 $\alpha$-positive cells observed in the necrotic area. However, positive cells were apparent in Group IV, which indicated that
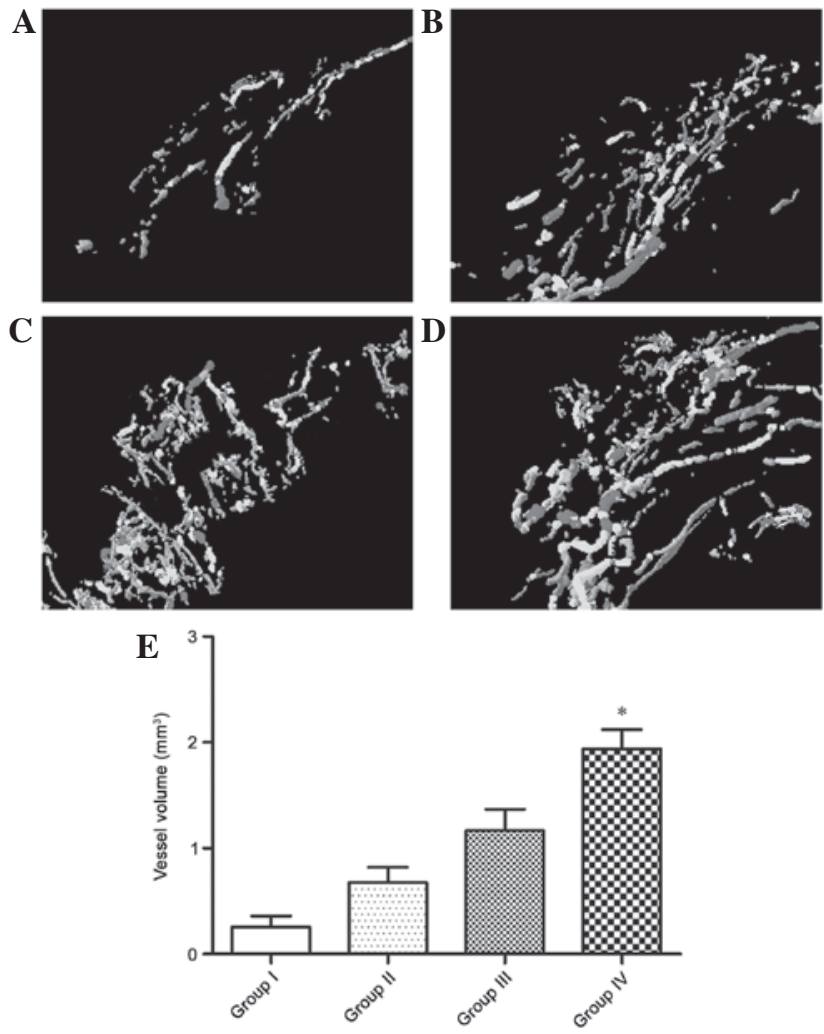

Figure 4. Assessment of neovascularization in the necrotic area of various groups. Representative images of micro-CT reconstructed three-dimensional microangiography of the necrotic area in groups (A) I, (B) II, (C) III and (D) IV. (E) Quantitative analysis of micro-CT data showing the newly formed vessel volume in the necrotic area of Group IV was significantly increased compared with the other three groups $\left({ }^{*} \mathrm{P}<0.05\right)$. CT, computed tomography.

sustained release of DMOG could enhance the expression of HIF-1 $\alpha$ in ASCs in vivo.

\section{Discussion}

Corticosteroid-induced ONFH is a major type of ONFH, which is a serious complication of corticosteroid treatment for autoimmune diseases, such as systemic lupus erythematosus, nephrotic syndrome and rheumatoid arthritis (27). The diagnostic or therapeutic strategy for ONFH is best introduced at the early stage before the disease becomes irreversible. Currently, the treatment methods of early-stage ONFH are highly controversial (28). Core decompression is reported to be effective for early-stage $\mathrm{ONFH}$, and acts to reverse the natural process of ONFH by providing a conduit for angiogenesis to revascularize subchondral bone and reducing elevated intraosseous pressures $(29,30)$. However, the majority of studies about the effectiveness of core decompression suggest that the results of this approach are not satisfactory $(31,32)$.

The aseptic necrotic bone in the femoral head retains its normal strength until the natural process of revascularization starts to remove the dead bone in preparation for the formation of new bone. As repair advances, the speed of angiogenesis and osteogenesis becomes slower than absorption of the dead bone, which may cause a weakening of mechanical structure (3). Jones hypothesized that new capillaries could only penetrate $10-15 \mathrm{~mm}$ of necrotic bone, and subchondral bone tissue 

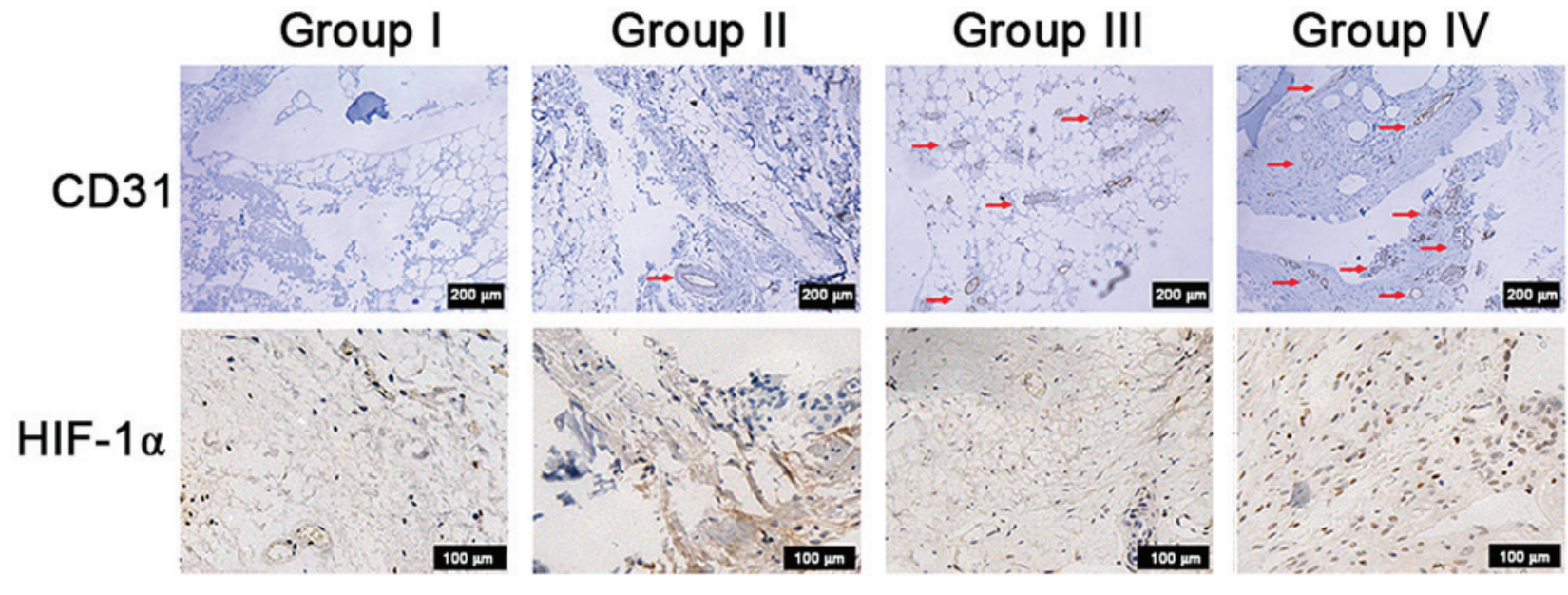

Figure 5. Immunohistochemistry for CD31 and HIF-1 $\alpha$. There were nearly no CD31-positive vessels observed in Groups I and II, few CD31-positive vessels observed in Group III, and obviously increased CD31-positive vessels in Group IV. Red arrowheads indicate CD31-positive vessels. There were numerous HIF-1 $\alpha$-positive cells present in Group IV, while nearly no HIF-1 $\alpha$-positive cells in groups I, II and III. HIF-1 $\alpha$, hypoxia inducible factor-1 $\alpha$.

usually can not be repaired (33). Furthermore, microfractures of the subchondral bone may retard capillary penetration, and then the loaded areas would collapse (33). Thus, it is important for ONFH treatment to increase local vascularization and new bone formation.

It has been reported that the MSCs pool of the femoral head could not provide enough osteoblasts to meet the need of local bone regeneration (34). Researchers have also suggested that MSCs in the proximal femur have a decreased proliferative and differentiation capacity at the early stage of $\mathrm{ONFH}(35,36)$. Therefore, MSCs have been employed to aid core decompression to treat early-stage ONFH $(11,12,37)$. Hypoxia inducible factor-1a (HIF-1 $\alpha$ ) is an important functional subunit of the HIF family, which are crucial mediators of the adaptive cells response to hypoxia (17). HIF-1 $\alpha$ arose early in evolution, and is widely expressed in most human tissues (17). A previous study found that HIF-1 $\alpha$ controls the expression of numerous genes in cells, cell proliferation and differentiation (38). Our previous study showed that, compared with normal MSCs, HIF-1 $\alpha$ transgenic MSCs had improved osteogenic and angiogenic capacity in vitro and better potential to promote bone regeneration in the necrotic area of the femoral head (19).

However, the risks of gene transduction, such as tumorigenesis and permanent overexpression of the transduced gene, limited its clinical application (20). To overcome these deficiencies, in the present study dimethyloxaloylglycine (DMOG), a cell permeable prolyl-4-hydroxylase inhibitor, was employed to enhance HIF-1 $\alpha$ expression in MSCs in place of the HIF-1 $\alpha$ gene transduction.

DMOG is a small molecular drug, which is able to stabilize the expression of HIF-1 $\alpha$ in cells (21). At normal oxygen tension, HIF- $1 \alpha$ is modified by oxygen dependent prolyl hydroxylases, resulting in rapid degradation through the ubiquitin-proteasome pathway (22). Under hypoxic conditions, prolyl hydroxylase activity decreases and HIF-1 $\alpha$ is stabilized (39). DMOG is able to inhibit prolyl hydroxylase activity at normal oxygen tension, thus leading to HIF-1 $\alpha$ overexpression in cells. DMOG has been used to treat ischemic skeletal muscles, middle cerebral artery occlusion and ischemic myocardial injury, and all resulted in good outcomes in previous experiments (40-42). Our previous study showed that DMOG could significantly enhance VEGF production in ASCs and improved the osteogenic differentiation potential of ASCs in vitro by stabilizing the expression of HIF-1 $\alpha$ in ASCs, and DMOG-treated ASCs had an increased bone healing capacity and promoted local revascularization in critical defects (22). In the present study, we investigated the efficacy of implanting DMOG-treated ASCs to treat early-stage corticosteroid-induced ONFH.

This study used a rabbit model with steroid-induced early-stage ONFH. An animal model of ONFH is important for evaluation of the effects of various methods of treating ONFH. In the present model, osteonecrosis was induced by a combination of LPS and MPS to mimic the pathological mechanism of steroid-induced ONFH. It has been reported that this inductive protocol has a high incidence of $\mathrm{ONFH}$, and resulted in nearly no death, and suggested that numerous histopathological and pathogenetic features of steroid-induced ONFH in rabbits are similar to those observed in human early-stage ONFH (25). Therefore, the steroid-induced rabbit ONFH model may be effective for testing the efficacy of the method developed for treatment of steroid-associated ONFH for clinical application and confirmation of their long-term effects in prevention of subchondral bone collapse of the hips.

In the present study, DMOG-treated ASCs were implanted into the necrotic area of the femoral head to aid core decompression in improving bone healing. Micro-CT and histological results indicated there was increased bone regeneration in the normal ASCs group than in the group that underwent core decompression only, which suggests that ASCs could improve bone healing on their own. Increased newly formed bone was observed in the DMOG-treated ASCs group than in the normal ASCs group, which indicated DMOG could enhance the bone healing capacity of ASCs in the necrotic area. Micro-CT and immunohistochemistry detection of CD31 showed that vessels formation was also increased in the DMOG-treated ASCs group than in the other three groups. This may be because DMOG could increase the expression and secretion of certain angiogenic factors in ASCs in vivo by stabilizing the HIF-1 $\alpha$ expression in cells, which has been demonstrated by previous 
experiments (43). It is hypothesized that angiogenesis is closely associated with osteogenesis, and that neovascularization is an favorable element in bone regeneration. Therefore, the improved local blood supply may also contribute to bone healing in the necrotic area.

In summary, the results of the present study showed that DMOG-treated ASCs markedly increased vascularization and bone regeneration in necrotic area of the femoral head in ONFH rabbits. These findings suggest that DMOG could enhance the osteogenic activity of ASCs in vivo, and may therefore provide a novel and effective therapeutic option for early-stage corticosteroid-induced ONFH.

\section{Acknowledgements}

This study was supported by the National Natural Science Foundation of China (grant nos. 81272004, 81272003 and 81371962) and Foundation of Shanghai Sixth People's Hospital (grant no. 1631).

\section{References}

1. Kerachian MA, Harvey EJ, Cournoyer D, Chow TY and Séguin C: Avascular necrosis of the femoral head: Vascular hypotheses. Endothelium 13: 237-244, 2006.

2. Bachiller FG, Caballer AP and Portal LF: Avascular necrosis of the femoral head after femoral neck fracture. Clin Orthop Relat Res 87-109, 2002.

3. Lieberman JR, Berry DJ, Mont MA, Aaron RK, Callaghan JJ, Rajadhyaksha AD and Urbaniak JR: Osteonecrosis of the hip: Management in the 21st century. Instr Course Lect 52: 337-355, 2003.

4. Yoon TR, Song EK, Rowe SM and Park CH: Failure after core decompression in osteonecrosis of the femoral head. Int Orthop 24: 316-318, 2001.

5. Yuan J, Cui L, Zhang WJ, Liu W and Cao Y: Repair of canine mandibular bone defects with bone marrow stromal cells and porous beta-tricalcium phosphate. Biomaterials 28: 1005-1013, 2007.

6. Liu G, Zhao L, Zhang W, Cui L, Liu W and Cao Y: Repair of goat tibial defects with bone marrow stromal cells and beta-tricalcium phosphate. J Mater Sci Mater Med 19: 2367-2376, 2008.

7. Stamm C, Westphal B, Kleine HD, Petzsch M, Kittner C, Klinge $\mathrm{H}$, Schümichen $\mathrm{C}$, Nienaber $\mathrm{CA}$, Freund $\mathrm{M}$ and Steinhoff G: Autologous bone-marrow stem-cell transplantation for myocardial regeneration. Lancet 361: 45-46, 2003.

8. Schächinger V, Erbs S, Elsässer A, Haberbosch W, Hambrecht R, Hölschermann H, Yu J, Corti R, Mathey DG, Hamm CW, et al: Intracoronary bone marrow-derived progenitor cells in acute myocardial infarction. N Engl J Med 355: 1210-1221, 2006.

9. Dadon-Nachum M, Sadan O, Srugo I, Melamed E and Offen D: Differentiated mesenchymal stem cells for sciatic nerve injury. Stem Cell Rev 7: 664-671, 2011.

10. Yang Y, Hallgrimsson B and Putnins EE: Craniofacial defect regeneration using engineered bone marrow mesenchymal stromal cells. J Biomed Mater Res A 99: 74-85, 2011.

11. Gangji V, De Maertelaer V and Hauzeur JP: Autologous bone marrow cell implantation in the treatment of non-traumatic osteonecrosis of the femoral head: Five year follow-up of a prospective controlled study. Bone 49: 1005-1009, 2011.

12. Tzaribachev N, Vaegler M, Schaefer J, Reize P, Rudert M, Handgretinger R and Müller I: Mesenchymal stromal cells: A novel treatment option for steroid-induced avascular osteonecrosis. Isr Med Assoc J 10: 232-234, 2008.

13. Cowan CM, Shi YY, Aalami OO, Chou YF, Mari C, Thomas R, Quarto N, Contag CH, Wu B and Longaker MT: Adipose-derived adult stromal cells heal critical-size mouse calvarial defects. Nat Biotechnol 22: 560-567, 2004.

14. Dudas JR, Marra KG, Cooper GM, Penascino VM, Mooney MP, Jiang S, Rubin JP and Losee JE: The osteogenic potential of adipose-derived stem cells for the repair of rabbit calvarial defects. Ann Plast Surg 56: 543-548, 2006.
15. Nikol S, Engelmann MG, Pelisek J, Fuchs A, Golda A, Shimizu M, Mekkaoui C and Rolland PH: Local perivascular application of low amounts of a plasmid encoding for vascular endothelial growth factor (VEGF165) is efficient for therapeutic angiogenesis in pigs. Acta Physiol Scand 176: 151-159, 2002.

16. Elçin YM, Dixit V and Gitnick G: Extensive in vivo angiogenesis following controlled release of human vascular endothelial cell growth factor: Implications for tissue engineering and wound healing. Artif Organs 25: 558-565, 2001

17. Mazumdar J, Dondeti V and Simon MC: Hypoxia-inducible factors in stem cells and cancer. J Cell Mol Med 13: 4319-4328, 2009.

18. Zou D, Zhang Z, Ye D, Tang A, Deng L, Han W, Zhao J, Wang S, Zhang W, Zhu C, et al: Repair of critical-sized rat calvarial defects using genetically engineered bone marrow-derived mesenchymal stem cells overexpressing hypoxia-inducible factor-1 $\alpha$. Stem Cells 29: 1380-1390, 2011.

19. Ding H, Gao YS, Hu C, Wang Y, Wang CG, Yin JM, Sun Y and Zhang CQ: HIF-1 $\alpha$ transgenic bone marrow cells can promote tissue repair in cases of corticosteroid-induced osteonecrosis of the femoral head in rabbits. PLoS One 8: e63628, 2013.

20. Cesana D, Ranzani M, Volpin M, Bartholomae C, Duros C, Artus A, Merella S, Benedicenti F, Sergi Sergi L, Sanvito F, et al: Uncovering and dissecting the genotoxicity of self-inactivating lentiviral vectors in vivo. Mol Ther 22: 774-785, 2014.

21. Jaakkola P, Mole DR, Tian YM, Wilson MI, Gielbert J, Gaskell SJ, von Kriegsheim A, Hebestreit HF, Mukherji M, Schofield CJ, et al: Targeting of HIF-alpha to the von Hippel-Lindau ubiquitylation complex by O2-regulated prolyl hydroxylation. Science 292: 468-472, 2001

22. Ding H, Gao YS, Wang Y, Hu C, Sun Y and Zhang C: Dimethyloxaloylglycine increases the bone healing capacity of adipose-derived stem cells by promoting osteogenic differentiation and angiogenic potential. Stem Cells Dev 23: 990-1000, 2014.

23. National Research Council Institute for Laboratory Animal Research: Guide for the Care and Use of Laboratory Animals. National Academies Press, Washington, DC, 1996.

24. Ding H, Chen S, Yin JH, Xie XT, Zhu ZH, Gao YS and Zhang CQ: Continuous hypoxia regulates the osteogenic potential of mesenchymal stem cells in a time-dependent manner. Mol Med Rep 10: 2184-2190, 2014.

25. Qin L, Zhang G, Sheng H, Yeung KW, Yeung HY, Chan CW, Cheung WH, Griffith J, Chiu KH and Leung KS: Multiple bioimaging modalities in evaluation of an experimental osteonecrosis induced by a combination of lipopolysaccharide and methylprednisolone. Bone 39: 863-871, 2006.

26. Sun Y, Feng $Y$ and Zhang C: The effect of bone marrow mononuclear cells on vascularization and bone regeneration in steroid-induced osteonecrosis of the femoral head. Joint Bone Spine 76: 685-690, 2009.

27. Griffith JF, Antonio GE, Kumta SM, Hui DS, Wong JK, Joynt GM, Wu AK, Cheung AY, Chiu KH, Chan KM, et al: Osteonecrosis of hip and knee in patients with severe acute respiratory syndrome treated with steroids. Radiology 235: 168-175, 2005.

28. Ohzono K, Saito M, Sugano N, Takaoka K and Ono K: The fate of nontraumatic avascular necrosis of the femoral head. A radiologic classification to formulate prognosis. Clin Orthop Relat Res 277: 73-78, 1992.

29. Hopson CN and Siverhus SW: Ischemic necrosis of the femoral head. Treatment by core decompression. J Bone Joint Surg Am 70: 1048-1051, 1988.

30. Maniwa S, Nishikori T, Furukawa S, Kajitani K, Iwata A, Nishikawa $U$ and Ochi M: Evaluation of core decompression for early osteonecrosis of the femoral head. Arch Orthop Trauma Surg 120: 241-244, 2000.

31. Dailiana ZH, Toth AP, Gunneson E, Berend KR and Urbaniak JR: Free vascularized fibular grafting following failed core decompression for femoral head osteonecrosis. J Arthroplasty 22: 679-688, 2007.

32. Scully SP, Aaron RK and Urbaniak JR: Survival analysis of hips treated with core decompression or vascularized fibular grafting because of avascular necrosis. J Bone Joint Surg Am 80: 1270-1275, 1998

33. Jones JP Jr: Concepts of etiology and early pathogenesis of osteonecrosis. Instr Course Lect 43: 499-512, 1994.

34. Hernigou P, Beaujean F and Lambotte JC: Decrease in the mesenchymal stem-cell pool in the proximal femur in corticosteroid-induced osteonecrosis. J Bone Joint Surg Br 81: 349-355, 1999. 
35. Gangji V, Hauzeur JP, Schoutens A, Hinsenkamp M, Appelboom T and Egrise D: Abnormalities in the replicative capacity of osteoblastic cells in the proximal femur of patients with osteonecrosis of the femoral head. J Rheumatol 30: 348-351, 2003.

36. Lee JS, Lee JS, Roh HL, Kim CH, Jung JS and Suh KT: Alterations in the differentiation ability of mesenchymal stem cells in patients with nontraumatic osteonecrosis of the femoral head: Comparative analysis according to the risk factor. J Orthop Res 24: 604-609, 2006.

37. Yan ZQ, Chen YS, Li WJ, Yang Y, Huo JZ, Chen ZR, Shi JH and Ge JB: Treatment of osteonecrosis of the femoral head by percutaneous decompression and autologous bone marrow mononuclear cell infusion. Chin J Traumatol 9: 3-7, 2006.

38. Riddle RC, Khatri R, Schipani E and Clemens TL: Role of hypoxia-inducible factor-1alpha in angiogenic-osteogenic coupling. J Mol Med (Berl) 87: 583-590, 2009.

39. Piret JP, Lecocq C, Toffoli S, Ninane N, Raes M and Michiels C: Hypoxia and $\mathrm{CoCl}_{2}$ protect HepG2 cells against serum deprivation- and t-BHP-induced apoptosis: A possible anti-apoptotic role for HIF-1. Exp Cell Res 295: 340-349, 2004.
40. Ockaili R, Natarajan R, Salloum F, Fisher BJ, Jones D, Fowler AA III and Kukreja RC: HIF-1 activation attenuates postischemic myocardial injury: Role for heme oxygenase-1 in modulating microvascular chemokine generation. Am J Physiol Heart Circ Physiol 289: H542-H548, 2005.

41. Milkiewicz M, Pugh CW and Egginton S: Inhibition of endogenous HIF inactivation induces angiogenesis in ischaemic skeletal muscles of mice. J Physiol 560: 21-26, 2004.

42. Nagel S, Papadakis M, Chen R, Hoyte LC, Brooks KJ, Gallichan D, Sibson NR, Pugh C and Buchan AM: Neuroprotection by dimethyloxalylglycine following permanent and transient focal cerebral ischemia in rats. J Cereb Blood Flow Metab 31: 132-143, 2011.

43. Ding H, Chen S, Song WQ, Gao YS, Guan JJ, Wang Y, Sun Y and Zhang CQ: Dimethyloxaloylglycine improves angiogenic activity of bone marrow stromal cells in the tissue-engineered bone. Int J Biol Sci 10: 746-756, 2014. 